\title{
Quantification of bovine leukemia virus proviral DNA using a low-cost real-time polymerase chain reaction
}

\author{
M. I. Petersen, ${ }^{*}$ I. Alvarez, ${ }^{\star} \dagger$ K. G. Trono, ${ }^{*} \dagger$ and J. P. Jaworski ${ }^{*} \dagger^{1}$ \\ ${ }^{*}$ Consejo Nacional de Investigaciones Científicas y Tecnológicas, Buenos Aires, Argentina, 1425 \\ †Instituto Nacional de Tecnología Agropecuaria, Instituto de Virología, Buenos Aires, Argentina, 1686
}

\begin{abstract}
The detection of bovine leukemia virus (BLV) proviral DNA is an important tool to address whether an animal is infected with BLV. Compared with serological assays, real-time PCR accounts for greater sensitivity and can serve as a confirmatory test for the clarification of inconclusive or discordant serological test results. However, the high cost related to real-time PCR assays has limited their systematic inclusion in BLV surveillance and eradication programs. The aim of the present study was to validate a low-cost quantitative real-time PCR. Interestingly, by using SYBR Green detection dye, we were able to reduce the cost of a single reaction by a factor of 5 compared with most common assays based on the use of fluorogenic probes (i.e., TaqMan technology). This approach allowed a highly sensitive and specific detection and quantification of BLV proviral DNA from purified peripheral blood leukocytes and a milk matrix. Due to its simplicity and low cost, our in-house BLV SYBR quantitative real-time PCR might be used either as a screening or as a confirmatory test in BLV control programs.
\end{abstract}

Key words: bovine leukemia virus, proviral DNA, real-time polymerase chain reaction, epidemiology

\section{INTRODUCTION}

Bovine leukemia virus (BLV) is a deltaretrovirus from the Orthoretrovirinae subfamily and Retroviridae family. The BLV causes a persistent infection in cattle, and in most cases this infection is asymptomatic (Ghysdael et al., 1984). In one-third of infected animals the infection progresses to a state of persistent lymphocytosis, and in 1 to $10 \%$ of infected cattle it develops into lymphosarcoma (Ghysdael et al., 1984). Bovine leukemia virus is distributed worldwide with the exception of Western Europe and Oceania. This

Received December 6, 2017.

Accepted March 1, 2018.

${ }^{1}$ Corresponding author: jaworski.juan@inta.gob.ar virus was first found in Argentina in 1973; since then, BLV rapidly spread throughout the region (Ciprian, 1973; Trono et al., 2001). A recent study highlighted that the average within-herd prevalence of BLV in the main productive area of Argentina was 80\% (Gutiérrez et al., 2012). High prevalence of BLV is associated with a significant economic impact on the dairy industry due to trade restrictions, replacement cost, reduced milk production, immunosuppression, and increased susceptibility to pneumonia, diarrhea, mastitis, and so on (Trainin et al., 1996; Bartlett et al., 2014; Frie and Coussens, 2015).

Classic BLV eradication programs rely on the correct identification and segregation or elimination of BLV-infected animals. Serologic assays for the detection of BLV-specific antibodies in sera and milk have been widely used as screening tests due to their high sensitivity and ability to test a large number of samples at a very low cost. In this regard, the agar gel immunodiffusion and ELISA are both cited as prescribed tests (OIE, 2012). However, serologic tests might fail to detect BLV-specific antibodies in samples from animals that have been recently infected with BLV due to the low level of BLV-specific antibodies present in these particular samples (Eaves et al., 1994). Furthermore, the inclusion of highly sensitive screening assays might increase the efficiency of a particular eradication program, especially for herds with a low within-herd prevalence. For that reason, the development and validation of more sensitive assays for BLV testing in cattle are necessary.

Nucleic acid amplification tests, such as PCR, allow a rapid and highly sensitive detection of BLV proviral DNA, even during recent infection. Compared with serological assays, quantitative real-time PCR (qPCR) not only allows the detection of BLV infection but also allows the estimation of BLV proviral load, which directly correlates with the disease stage and the risk of disease transmission (Yuan et al., 2015; Juliarena et al., 2016). Additionally, qPCR assays can serve as confirmatory tests for the clarification of inconclusive and discordant serological test results (Rola-Łuszczak et al., 
Table 1. Bovine leukemia virus (BLV) quantitative real-time PCR feasibility and reproducibility using a reference panel

\begin{tabular}{|c|c|c|c|c|c|c|}
\hline $\begin{array}{l}\text { Sample ID } \\
{(\text { BLV status })^{1}}^{1}\end{array}$ & $\begin{array}{c}\text { BLV copies, }{ }^{2} \\
\text { no. } / \mu \mathrm{g} \text { of DNA }\end{array}$ & $\begin{array}{l}\mathrm{Ct}^{3} \\
\text { mean }\end{array}$ & SD & $\begin{array}{c}\mathrm{CV} \\
\%\end{array}$ & $\begin{array}{c}\text { Replicates, } \\
\text { no. }\end{array}$ & $\begin{array}{l}\text { Positive } \\
\text { replicates, no. }\end{array}$ \\
\hline$\overline{\mathrm{A}(-)}$ & $\mathrm{NA}^{4}$ & 0 & 0.0 & NA & 8 & 0 \\
\hline $\mathrm{B}(-)$ & NA & 0 & 0.0 & NA & 8 & 0 \\
\hline $\mathrm{C}(+)$ & 45,000 & 22.74 & 0.28 & 1.23 & 8 & 8 \\
\hline $\mathrm{D}(+)$ & 10,000 & 23.61 & 0.09 & 0.40 & 8 & 8 \\
\hline $\mathrm{E}(+)$ & 8,000 & 24.25 & 0.08 & 0.35 & 8 & 8 \\
\hline $\mathrm{F}(+)$ & 2,000 & 26.39 & 0.10 & 0.37 & 8 & 8 \\
\hline $\mathrm{G}(+)$ & 1,000 & 28.55 & 0.19 & 0.66 & 8 & 8 \\
\hline $\mathrm{H}(+)$ & 500 & 28.88 & 0.32 & 1.10 & 8 & 8 \\
\hline $\mathrm{I}(+)$ & 500 & 29.00 & 0.41 & 1.40 & 8 & 8 \\
\hline $\mathrm{J}(+)$ & 400 & 29.02 & 0.14 & 0.47 & 8 & 8 \\
\hline $\mathrm{K}(+)$ & 400 & 29.92 & 0.22 & 0.72 & 8 & 8 \\
\hline $\mathrm{L}(+)$ & 10 & 30.71 & 0.67 & 2.18 & 8 & 8 \\
\hline $\mathrm{M}^{\prime}(+)$ & 100 & 31.05 & 0.44 & 1.41 & 8 & 8 \\
\hline $\mathrm{N}(+)$ & 50 & 31.64 & 0.71 & 2.25 & 8 & 8 \\
\hline
\end{tabular}

2013). Several $\mathrm{qPCR}$ assays have been developed for BLV proviral DNA detection (Lew et al., 2004; Heenemann et al., 2012; Rola-Łuszczak et al., 2013). Previous studies comparing pol, gag, and env genes reported that pol was the most suitable gene to target for detection purposes because it provided the most sensitive assays (Rola-Łuszczak et al., 2013). This might be in part due to a higher sequence conservation of pol among strains from different geographical areas. Importantly, most of these assays are based on the use of fluorogenic probes, making them very expensive for use as screening tests in BLV eradication programs.

The aim of the present study was to validate a low-cost qPCR for the detection of BLV proviral DNA. For that purpose we used an international panel of BLV proviral DNA samples and a set of field samples obtained from the main productive area of Argentina. Overall, the assay allowed a highly sensitive and specific detection of BLV proviral DNA from purified peripheral blood leukocytes (PBL) and a milk matrix.

\section{MATERIALS AND METHODS}

\section{Reference Samples}

Bovine leukemia virus DNA reference samples were obtained from 12 serologically positive and 2 serologically negative cattle from 7 countries (Ukraine, Russia, Moldova, Croatia, Japan, Argentina, and Poland). The number of BLV proviral DNA copies in each of these samples was determined by qPCR in 5 labora- tories worldwide: (1) the Molecular and Cellular Biology Laboratory of Gemboux, University of Liege, Liege, Belgium; (2) the Institute of Virology, Center for Infectious Diseases, University of Leipzig, Leipzig, Germany; (3) the Department of Veterinary Medicine, Iwate University, Iwate, Japan; (4) the National Veterinary Research Institute (NVRI), Pulawy, Poland; and (5) the Animal and Plant Health Agency, Weybridge, United Kingdom. Three laboratories at the Animal and Plant Health Agency, NVRI, and Leipzig University act as the World Organization of Animal Health reference laboratories for enzootic bovine leukemia. The average number of BLV copies in these samples ranged from 10 to $50,000 / \mu \mathrm{g}$ of total DNA (Table 1 ). The reference panel was kindly provided by Jacek Kuzmak (NVRI, Poland).

\section{Field Samples Collection and DNA Extraction}

Field samples were obtained from a dairy farm located in Rafaela, Santa Fe, Argentina $\left(31^{\circ} 16 \mathrm{~S}, 61^{\circ} 29 \mathrm{~W}\right)$. This region belongs to the main dairy-producing area of the country, with an average within-herd prevalence of BLV of $80 \%$. The farm comprised a milking herd ( $\mathrm{n}$ $=330$ Holstein cows), 64 dry cows, 94 heifers $(>1$ yr) and 123 calves $(<1 \mathrm{yr}$ ). The BLV serological status of all animals was determined by ELISA at the time that the study was initiated. Consistent with other farms located in the same region, the individual cow prevalence for this particular farm was close to $90 \%$. For this study, a total of 67 animals ( $>1 \mathrm{yr}$ ) were randomly selected 
based on their serological status (24 BLV sero-negative and $43 \mathrm{BLV}$ sero-positive animals). Sero-conversion was investigated in all negative animals $60 \mathrm{~d}$ after the original screening. The procedures used for animal handling and sampling were approved by the Institutional Animal Care and Use Committee of the INTA. The guidelines described in the institutional manual were followed at all times. Peripheral blood leukocytes were isolated from field samples by centrifugation at 1,500 $\times g$ for $25 \mathrm{~min}$, and erythrocytes were hemolysed by osmotic shock with $\mathrm{H}_{2} \mathrm{O}$ and $4.5 \% \mathrm{NaCl}$. After 2 washes in PBS, the supernatant was discarded and the cell pellet was used for extraction of genomic DNA. The genomic DNA was extracted using High Pure PCR Template Preparation Kit (Roche, Penzberg, Germany) according to the manufacturer's instructions. The DNA concentration was calculated using a nanophotometer (Nanodrop, Thermo Fisher Scientific, Waltham, MA). The DNA samples were stored at $-80^{\circ} \mathrm{C}$ until use.

\section{Cost Analysis of BLV Real-Time qPCR}

TaqMan versus SYBR Green (both now from Thermo Fisher Scientific) dye detection system costs were compared as part of the cost analysis. In this analysis, we considered the prices of reagents and consumables included in each of these qPCR reactions. Additionally, we focused on those supplies that we have access to in Argentina and those that are regularly purchased by our laboratory. Costs related to personnel, equipment, and facilities were not included in the present analysis. The cost associated with the extraction method was not considered either.

\section{BLV SYBR Green Real-Time qPCR}

The original BLV qPCR protocol described by RolaEuszczak et al. (2013) was adapted to be used with an SYBR Green dye detection system. Briefly, each BLV SYBR qPCR reaction contained Fast Start Universal SYBR Green Master Mix (Roche), $800 \mathrm{n} M$ forward and reverse primers (BLV pol 5f: 5'-CCTCAATTCCCTTTAAACTA-3'; BLV pol3r: 5'-GTACCGGGAAGACTGGATTA-3'), and $500 \mathrm{ng}$ of DNA template. The reaction was performed on an ABI 7500 machine (Thermo Fisher Scientific) with the following cycling conditions: 2 min at $50^{\circ} \mathrm{C} ; 95^{\circ} \mathrm{C}$ for $10 \mathrm{~min}$; followed by 40 cycles of $95^{\circ} \mathrm{C}$ for $15 \mathrm{~s}, 55^{\circ} \mathrm{C}$ for $15 \mathrm{~s}$, and at $60^{\circ} \mathrm{C}$ for $1 \mathrm{~min}$. As standard, the plasmid pBLV1 (kindly provided by Jacek Kuzmak, NVRI) containing BLV pol fragment was used. Ten-fold dilutions of this standard were made from $5 \times 10^{6}$ copies $/ \mu \mathrm{L}$ to $5 \times 10^{0}$ copy $/ \mu \mathrm{L}$. A strong and a weak positive control as well as a negative control and a no-template control were included in each plate.

\section{Validation of BLV SYBR qPCR}

Feasibility, Reproducibility, and Analytical Sensitivity of BLV SYBR $q P C R$. To determine the feasibility of our in-house BLV SYBR qPCR, we analyzed 14 DNA samples from the reference panel (12 BLV-positive and $2 \mathrm{BLV}$-negative samples). These samples were tested in duplicate in 4 different runs of the assay. The specificity of each positive reaction was confirmed by dissociation curve analysis [melting temperature $\left.\left(\mathbf{T}_{\mathbf{m}}\right)_{\text {pol amplicon }}=81.0 \pm 1.0^{\circ} \mathrm{C}\right]$. In addition, all samples amplified by BLV SYBR qPCR were assayed by electrophoresis in agarose gel to confirm these results.

The preliminary reproducibility of the BLV SYBR qPCR was determined based on the level of variability of the cycle thresholds (Ct) obtained for different replicates of each reference sample. To determine the analytical sensitivity of the BLV SYBR qPCR, we selected the sample with the lowest $\mathrm{Ct}$ from the reference panel (sample C from Table 1). Based on the information provided by the reference laboratory regarding BLV copy numbers, 10 -fold serial dilutions of this sample were performed from $2 \times 10^{3}$ to $2 \times 10^{-1}$ copies of BLV proviral DNA per reaction. These series of dilutions were assayed by BLV SYBR qPCR in 8 replicates.

Standard Curve for Quantification of $B L V$ Pol Copy Number. A plasmid containing BLV pol fragment (pBLV1) was used as standard. The plasmid pBLV1 was kindly provided by Jacek Kuzmak at NVRI. To build a standard curve, 10-fold serial dilutions of the plasmid pBLV1 were performed from 5 $\times 10^{6}$ to $5 \times 10^{0}$ copies per reaction. Except for the dilution $5 \times 10^{6}$, which was tested in 16 replicates, all the dilutions were tested in 36 replicates (2 replicates per run in 18 runs of the assay). The reproducibility of the BLV SYBR qPCR was estimated by analyzing the coefficient of variation $(\mathbf{C V})$ for each point of the standard curve. The efficiency, coefficient of determination, and dynamic range of the BLV SYBR qPCR were also estimated.

Efficiency of BLV SYBR qPCR Using a Milk Matrix. To test the efficiency of the BLV SYBR qPCR in a milk matrix, the standard pBLV1 was 10 -fold serially diluted in bulk milk from uninfected cows. For this purpose we pooled individual milk samples from 3 BLV sero-negative animals from the same herd. The BLV status in these animals was confirmed by testing individual plasma and milk samples by BLV qPCR. Then, the total DNA from each dilution was purified by testing individual blood and milk samples using a High Pure PCR Template Preparation Kit (Roche) according to the manufacturer's instructions. Each fraction was assayed in 8 replicates from 4 independent runs by 
BLV SYBR qPCR. The analytical sensitivity of BLV SYBR qPCR in this milk matrix was also tested.

Diagnostic Performance of $B L V \quad S Y B R$ $\boldsymbol{q P C R}$. A total of 67 field samples (described above) were tested to evaluate the diagnostic sensitivity and specificity of the BLV SYBR qPCR. The BLV status in these animals was confirmed by BLV nested PCR and the detection of BLV-specific antibodies in plasma by ELISA (see description of these assays below). Additionally, the analytical specificity of the BLV SYBR $\mathrm{qPCR}$ was determined through the analysis of several cell lines infected with other viruses that frequently cause infections in cattle, such as enterovirus, bovine herpesvirus, bluetongue virus, and bovine respiratory syncytial virus.

\section{BLV Nested PCR}

The BLV nested PCR (nPCR) was adapted from Kerkhofs et al. (1998). Briefly, the first round of the reaction contained $5 \times$ Colorless GoTaq Buffer (Promega, Fitchburg, WI), 0.5 U of Taq Polymerase (Promega), 5 $\mathrm{m} M$ of each deoxynucleotide triphosphate (Promega), $350 \mathrm{n} M$ of each primer (outer forward: $5^{\prime}$-ctcacttctgcttcaccatcc-3'; outer reverse: 5'-ggcaggcatgtagagagtgg- $\left.3^{\prime}\right)$, and 100 to $1,000 \mathrm{ng}$ of DNA template in a total reaction volume of $25 \mu \mathrm{L}$. The first reaction was performed with the following cycling conditions: $5 \mathrm{~min}$ at $95^{\circ} \mathrm{C}$; followed by 20 cycles at $95^{\circ} \mathrm{C}$ for $1 \mathrm{~min}, 61^{\circ} \mathrm{C}$ for $1 \mathrm{~min}$, and $72^{\circ} \mathrm{C}$ for $1 \mathrm{~min}$; followed by a last extension of $72^{\circ} \mathrm{C}$ for $5 \mathrm{~min}$. The second round of the reaction contained $5 \times$ Green GoTaq Buffer (Promega), $0.5 \mathrm{U}$ of Taq Polymerase (Promega), $5 \mathrm{~m} M$ deoxynucleotide triphosphates (Promega), $350 \mathrm{n} M$ of each primer (inner forward: 5'-tggaaagaactaacgctgacgg-3'; inner reverse: $5^{\prime}$-ccccaaccaacaacacttgctt- $3^{\prime}$ ), and $1 \mu \mathrm{L}$ of the product from the first round in a total volume of $25 \mu \mathrm{L}$. The conditions for this second round were 5 min at $95^{\circ} \mathrm{C}$; followed by 35 cycles at $95^{\circ} \mathrm{C}$ for $45 \mathrm{~s}, 65^{\circ} \mathrm{C}$ for $45 \mathrm{~s}$, and $72^{\circ} \mathrm{C}$ for $1 \mathrm{~min}$; followed by a last extension of $72^{\circ} \mathrm{C}$ for 5 min. The presence of BLV proviral DNA was evidenced by visualization of a 610 -bp fragment corresponding to the $p X$ region of the BLV genome. Positive and negative internal controls as well as nontemplate controls (for each round of reaction) were included in each run of the assay

\section{ELISA for the Detection of Anti-BLV Antibodies}

Plasma-specific antibodies against the whole BLV viral particle were measured by indirect ELISA as described previously (Trono et al., 2001). Briefly, ELISA plates were coated with antigen, from fetal lamb kidney cells persistently infected with BLV, purified by centrifugation on a sucrose cushion. The samples to be tested were added to the plate in duplicate. Based on preliminary data, plasma samples were prediluted 1:40. After incubation and washing, anti-bovine $\operatorname{IgG}$ peroxidase conjugated was added to each well. The presence of secondary antibody was revealed with $3^{\prime}$, $3^{\prime}, 5^{\prime}, 5$ '; -tetramethylbenzidine and $\mathrm{H}_{2} \mathrm{O}_{2}$. Reaction was stopped using $\mathrm{H}_{2} \mathrm{SO}_{4}$, and the absorbance was read at $450 \mathrm{~nm}$. Normalized results were obtained as a sample to positive ratio. A weak positive control serum was used to calculate the ratio. The difference between the raw OD obtained for the weak positive control and a negative control was set to $100 \%$. All tested samples were referred to it. A cut-off level of $25 \%$ was established in the original work over 339 serum samples using PCR and southern blot as confirmatory tests (Trono et al., 2001). The sensitivity and specificity of the assay were of 97.2 and 97.5 , respectively. Those samples with reactivity above the cut-off level were considered positive.

\section{RESULTS}

\section{Cost Analysis of BLV qPCR}

We compared costs of 2 detection systems extensively used in qPCR: TaqMan and SYBR Green (Supplemental Table S1; https://doi.org/10.3168/jds.2017-14253). By using the SYBR Green detection dye in our inhouse $\mathrm{BLV} \mathrm{qPCR}$, we reduced the cost per reaction from US\$3.11 (TaqMan detection system) to US $\$ 0.71$ (SYBR Green dye).

\section{Feasibility, Reproducibility, and Analytical Sensitivity of BLV SYBR qPCR}

Our in-house BLV SYBR qPCR detected true positive $(\mathrm{n}=12)$ and negative $(\mathrm{n}=2)$ samples from an international reference panel with a $100 \%$ concordance (Table 1). When dissociation curves analysis was performed on these samples, we observed that all positive samples exhibited the same melting temperature $\left(T_{m}\right.$ $=81.0 \pm 1.0^{\circ} \mathrm{C}$ ). Then, the products from BLV SYBR qPCR were assessed by electrophoresis in agarose gel. In this opportunity, all positive samples showed a specific band of $120 \mathrm{bp}$, confirming that amplification consisted of target sequence (data not shown). In contrast, no band was observed when BLV SYBR qPCR-negative samples were analyzed by gel electrophoresis.

Furthermore, we analyzed the level of variability of $\mathrm{Ct}$ among replicates of each reference sample to determine the preliminary reproducibility of the assay. The $\mathrm{CV}$ obtained for all $12 \mathrm{BLV}$-positive samples ranged from 0.35 to $2.25 \%$. 
Table 2. Bovine leukemia virus (BLV) quantitative real-time PCR (qPCR) analytical sensitivity using a reference sample

\begin{tabular}{|c|c|c|c|c|c|c|c|}
\hline \multirow[b]{2}{*}{ Copies } & \multicolumn{5}{|c|}{ BLV qPCR } & \multicolumn{2}{|c|}{ BLV nested PCR } \\
\hline & $\begin{array}{c}\mathrm{Ct}^{1} \\
\text { mean }\end{array}$ & $\mathrm{SD}$ & $\begin{array}{c}\mathrm{CV}, \\
\%\end{array}$ & $\begin{array}{l}\text { Replicates, } \\
\text { no. }\end{array}$ & $\begin{array}{c}\text { Positive replicates, } \\
\text { no. }(\%)\end{array}$ & $\begin{array}{c}\text { Replicates, } \\
\text { no. }\end{array}$ & $\begin{array}{c}\text { Positive replicates, } \\
\text { no. }(\%)\end{array}$ \\
\hline $2 \times 10^{3}$ & 25.36 & 1.29 & 5.09 & 8 & $8(100)$ & 6 & $6(100)$ \\
\hline $2 \times 10^{2}$ & 29.00 & 1.15 & 3.96 & 8 & $8(100)$ & 6 & $6(100)$ \\
\hline $2 \times 10^{1}$ & 32.69 & 1.41 & 4.30 & 8 & $8(100)$ & 6 & $6(100)$ \\
\hline $2 \times 10^{0}$ & 34.3 & 1.87 & 5.46 & 8 & $4(50)$ & 6 & $2(33.3)$ \\
\hline $2 \times 10^{-1}$ & 0 & 0 & $\mathrm{NA}^{2}$ & 8 & $0(0)$ & 6 & $0(0)$ \\
\hline
\end{tabular}

${ }^{1}$ Cycle threshold.

${ }^{2}$ Not applicable.

With the aim of testing the analytical sensitivity of the BLV SYBR qPCR, we performed 10-fold serial dilutions of reference sample $\mathrm{C}$ (Table 1) from 2 $\times 10^{3}$ to $2 \times 10^{-1}$ copies of BLV proviral DNA per reaction. Positive results were obtained in $100 \%$ of the replicates from $2 \times 10^{1}$ dilution and higher (Table 2 ). At dilution corresponding to $2 \times 10^{0}$ copies 4 out of 8 (50\%) replicates were positive, and at lower dilutions $\left(2 \times 10^{-1}\right.$ copies $)$ all replicates were negative. Thus, the sensitivity of the BLV SYBR qPCR was set at 2 copies per reaction. Additionally, to compare the analytical sensitivity of our BLV SYBR qPCR versus BLV nPCR, 10-fold serial dilutions of sample C from $2 \times 10^{3}$ to 2 $\times 10^{-1}$ copies of BLV proviral DNA per reaction were assayed by BLV nPCR. By nPCR, only 2 out of 6 replicates $(33 \%)$ corresponding to the dilution $2 \times 10^{0}$ were detected as positive (Table 2).

\section{Standard Curve for the Quantification of BLV pol Copy Number}

To build a standard curve, 10-fold serial dilutions of the plasmid pBLV1 ranging from $5 \times 10^{6}$ to $5 \times$ $10^{0}$ copies per reaction were assessed by BLV SYBR qPCR. Our BLV qPCR revealed positive results for all replicates at a dilution of $5 \times 10^{2}$ copies per reaction and higher (Table 3 ). At a dilution corresponding to 5 $\times 10^{1}$ and $5 \times 10^{0}$ copies, 33 out of $36(91.7 \%)$ and 24 out of $36(66.7 \%)$ replicates were positive, respectively. Additionally, the CV values increased as pBLV1 copies decreased; however, we observed a maximum variability $(\mathrm{CV}=5.06 \%)$ at a dilution of $5 \times 10^{1}$ copies. Overall, the efficiency of the qPCR reaction was close to $100 \%$ (slope $=-3.44$ ) and the dynamic range of the assay encompassed 6 orders of magnitude with a linear relationship $\left(R^{2}=0.996\right)$ between the $C t$ values and the standard DNA input copies (Figure 1). As noted in the following section, using this standard curve and within this dynamic range, we can assess BLV proviral loads from any sample by extrapolating the $\mathrm{Ct}$ values to BLV copy numbers per reaction.

\section{Efficiency of BLV SYBR qPCR Using a Milk Matrix}

With the aim of testing the efficiency and the analytical sensitivity of BLV SYBR qPCR in a milk matrix, 10-fold serial dilutions of pBLV1 standard in bulk milk from BLV-uninfected cows were assayed. Repeated testing of our assay revealed 20 copies of pBLV1 in $100 \%$ of replicates (8/8; Table 4). At a dilution corresponding to 2 copies, 5 out of 8 replicates were positive. Lower plasmid dilutions representing 0.2 copies of pBLV1 were consistently negative. Hence, the preliminary sensitivity of the BLV SYBR qPCR assay using a milk matrix was set at 2 copies of BLV proviral DNA. Using a milk matrix, our in-house BLV qPCR

Table 3. Bovine leukemia virus (BLV) qPCR analytical sensitivity using plasma BLV1 standard

\begin{tabular}{lccccc}
\hline $\begin{array}{l}\text { pBLV pol } \\
\text { copies }\end{array}$ & $\begin{array}{c}\mathrm{Ct}^{1} \\
\text { mean }\end{array}$ & $\mathrm{SD}$ & $\begin{array}{c}\mathrm{CV}, \\
\%\end{array}$ & $\begin{array}{c}\text { Replicates, } \\
\text { no. }\end{array}$ & $\begin{array}{c}\text { Positive replicates, } \\
\text { no. (\%) }\end{array}$ \\
\hline $5 \times 10^{6}$ & 14.85 & 0.20 & 1.36 & 16 & $16(100)$ \\
$5 \times 10^{5}$ & 17.05 & 0.46 & 2.69 & 36 & $36(100)$ \\
$5 \times 10^{4}$ & 20.33 & 0.49 & 2.41 & 36 & $36(100)$ \\
$5 \times 10^{3}$ & 23.77 & 0.71 & 3.01 & 36 & $36(100)$ \\
$5 \times 10^{2}$ & 27.52 & 0.83 & 3.03 & 36 & $33(92)$ \\
$5 \times 10^{1}$ & 31.22 & 1.58 & 5.06 & 36 & $24(67)$ \\
$5 \times 10^{0}$ & 34.10 & 0.98 & 2.89 & 36 & \\
\hline
\end{tabular}

${ }^{1}$ Cycle threshold. 
Table 4. Bovine leukemia virus (BLV) quantitative real-time PCR analytical sensitivity in a milk matrix using plasma BLV1

\begin{tabular}{lccccc}
\hline Copies & $\begin{array}{c}\mathrm{Ct}^{1} \\
\text { mean }\end{array}$ & SD & $\begin{array}{c}\text { CV, } \\
\%\end{array}$ & $\begin{array}{c}\text { Replicates, } \\
\text { no. }\end{array}$ & $\begin{array}{c}\text { Positive replicates, } \\
\text { no. (\%) }\end{array}$ \\
\hline $2 \times 10^{3}$ & 25.14 & 0.2 & 0.9 & 8 & $8(100)$ \\
$2 \times 10^{2}$ & 28.29 & 0.3 & 0.9 & 8 & $8(100)$ \\
$2 \times 10^{1}$ & 32.33 & 1.0 & 3.1 & 8 & $8(100)$ \\
$2 \times 10^{0}$ & 34.67 & 0.8 & 2.4 & 8 & $5(62)$ \\
$2 \times 10^{-1}$ & 0 & 0 & NA $^{2}$ & 8 & $0(0)$ \\
\hline
\end{tabular}

${ }^{1}$ Cycle threshold.

${ }^{2}$ Not applicable.

encompassed 4 orders of magnitude with a high linear relationship $\left(\mathrm{R}^{2}=0.987\right.$; data not shown). Moreover, low $\mathrm{CV}$ values observed at the different concentrations tested underscored a good reproducibility of the assay under these settings (Table 4).

\section{Diagnostic Performance of BLV SYBR qPCR}

A total of 67 field samples were tested by BLV SYBR qPCR, BLV nPCR, and ELISA, and results were compared among the different assays (Table 5). Twenty-three of these samples were negative and 41 were positive by all 3 techniques. From the remaining 3 samples, 1 (sample 24) was positive by our in-house
BLV SYBR qPCR and nPCR but negative by ELISA. To determine whether discordant results obtained for sample 24 were due to an unspecific PCR reaction or an incipient BLV infection with undetectable levels of BLV-specific antibody by ELISA, all 24 animals that initially tested negative by ELISA (the 23 negative by all 3 techniques plus the 1 negative by ELISA and positive by both PCR) were retested by ELISA 2 mo apart from the original screening time point. The seronegative status was confirmed in 23 out of 24 animals. In contrast, the animal that was initially negative by ELISA but positive by PCR (sample 24) seroconverted during that period of time. The second set of discordant samples (samples 25 and 26) were also positive

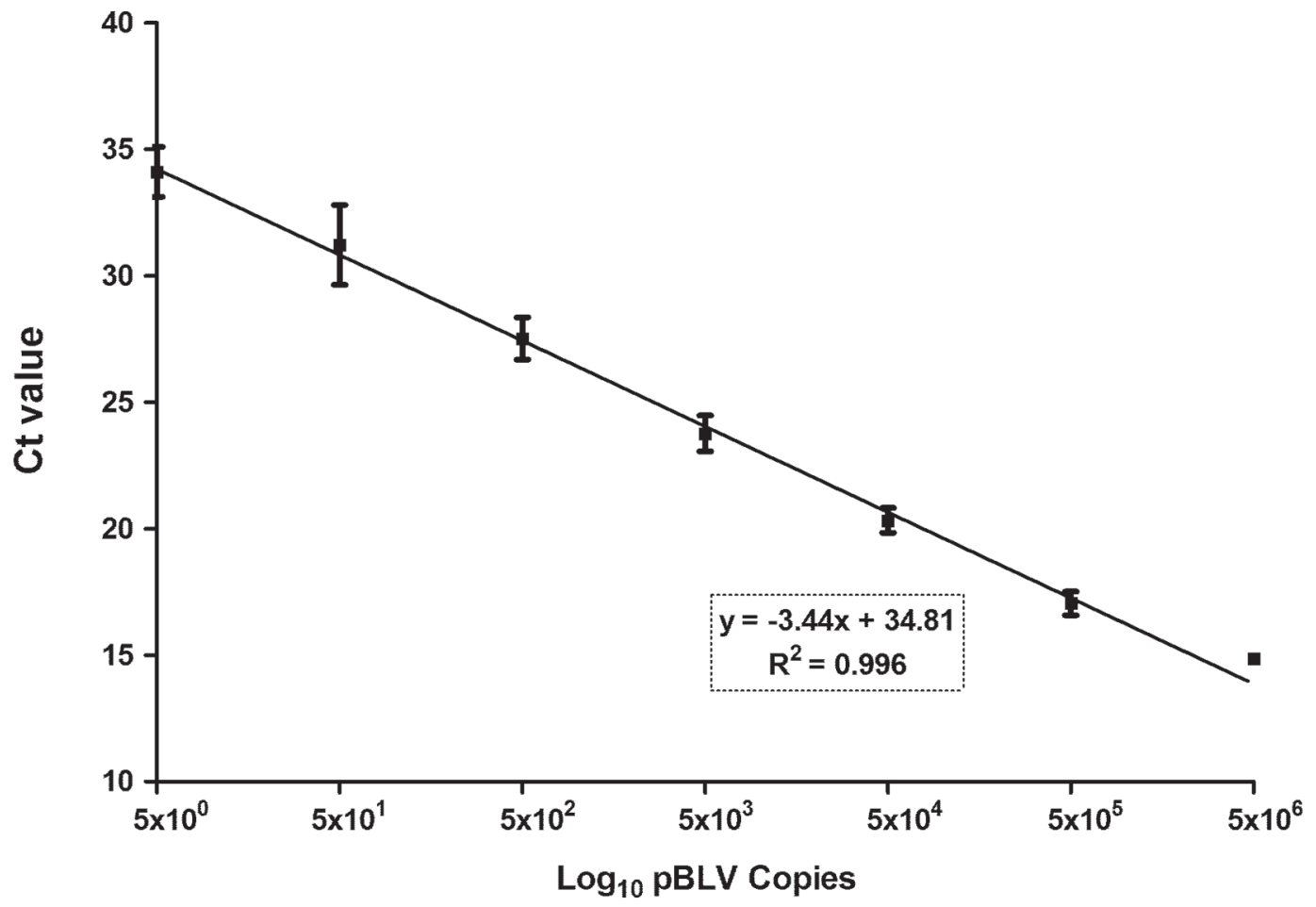

Figure 1. Standard curve for the quantification of bovine leukemia virus (BLV) pol copy number by BLV quantitative real-time PCR. Each dot represents the mean cycle threshold $(\mathrm{Ct})$ value of each dilution tested. pBLV = plasmid BLV. Error bars represent standard deviation. The slope and $\mathrm{R}^{2}$ values are represented in the figure. 
by ELISA and BLV SYBR qPCR but negative by BLV nPCR. Interestingly, the amount BLV proviral DNA in both samples was below the dynamic range of the BLV SYBR qPCR ( $<5$ copies per reaction). Additionally, to exclude the amplification of other viruses that frequently cause infections in cattle, cell lines infected with enterovirus, bovine herpesvirus, bluetongue virus, and bovine respiratory syncytial virus were investigated. None of these samples tested positive by BLV SYBR qPCR.

\section{DISCUSSION}

In the present study we validated a low-cost BLV SYBR qPCR that might be included as part of any eradication program in high-prevalence regions as well as in low- or mid-income countries. For that purpose, we modified the original protocol described by Kuzmak and colleagues and adapted it to be used with the SYBR Green detection system (Rola-Łuszczak et al., 2013). Interestingly, by using SYBR Green detection dye in our in-house BLV qPCR, we were able to reduce the cost of a single reaction by a factor of 5 compared with most common assays based on TaqMan technology. Remarkably, our BLV SYBR qPCR assay allowed a highly efficient, reproducible, sensitive, and specific detection and quantification of BLV proviral DNA from PBL and milk matrix.

Although qPCR assays based on SYBR Green dyes are in general less sensitive and specific than TaqManbased assays, we showed that our BLV SYBR qPCR displayed a high sensitivity. In this regard, our assay was able to detect as low as 5 copies per reaction of the plasmid pBLV1 (carrying BLV pol sequence) in $67 \%$ of tested replicates. In addition, based on the analysis of serial dilutions of a positive reference sample, the analytical sensitivity of our BLV SYBR qPCR assay was estimated in 2 BLV proviral DNA copies per reaction (detecting $50 \%$ of tested replicates). These observations are in accordance with previous reports by Rola-Łuszczak et al. (2013), in which the authors detected 1 copy of pBLV1 in 3 out of 5 replicates using the same set of primers but TaqMan technology. Moreover, the analytical sensitivity of our BLV SYBR qPCR was similar to the one obtained by nPCR (2 out of 6 positive replicates corresponding to a dilution of 2 copies per reaction). Interestingly, qPCR has several advantages compared with nPCR: (1) a shorter reaction time, (2) full automation, (3) results expressed as numbers, (4) no need for post-PCR processing, (5) less chance of cross-contamination, (6) collection of data in real time during the exponential growth phase, (7) an increased dynamic range of detection, and (8) the
Table 5. Diagnostic sensitivity and specificity of bovine leukemia virus (BLV) quantitative real-time PCR (qPCR) using field samples

\begin{tabular}{|c|c|c|c|c|c|}
\hline \multirow{2}{*}{$\begin{array}{l}\text { Sample } \\
\text { ID }\end{array}$} & \multirow[b]{2}{*}{ ELISA } & \multirow{2}{*}{$\begin{array}{c}\text { Nested } \\
\text { PCR }\end{array}$} & \multicolumn{3}{|c|}{ BLV qPCR } \\
\hline & & & Result & $\mathrm{Ct}^{1}$ mean & Copies/ $/ \mu \mathrm{g}$ of DNA \\
\hline 1 & - & - & - & $\mathrm{NA}^{2}$ & 0 \\
\hline 2 & - & - & - & NA & 0 \\
\hline 3 & - & - & - & NA & 0 \\
\hline 4 & - & - & - & NA & 0 \\
\hline 5 & - & - & - & NA & 0 \\
\hline 6 & - & - & - & NA & 0 \\
\hline 7 & - & - & - & NA & 0 \\
\hline 8 & - & - & - & NA & 0 \\
\hline 9 & - & - & - & NA & 0 \\
\hline 10 & - & - & - & NA & 0 \\
\hline 11 & - & - & - & NA & 0 \\
\hline 12 & - & - & - & NA & 0 \\
\hline 13 & - & - & - & NA & 0 \\
\hline 14 & - & - & - & NA & 0 \\
\hline 15 & - & - & - & NA & 0 \\
\hline 16 & - & - & - & $\mathrm{NA}$ & 0 \\
\hline 17 & - & - & - & NA & 0 \\
\hline 18 & - & - & - & NA & 0 \\
\hline 19 & - & - & - & NA & 0 \\
\hline 20 & - & - & - & NA & 0 \\
\hline 21 & - & - & - & NA & 0 \\
\hline 22 & - & - & - & NA & 0 \\
\hline 23 & - & - & - & NA & 0 \\
\hline 24 & - & + & + & 30.07 & 250 \\
\hline 25 & + & - & + & 38.07 & $<5$ \\
\hline 26 & + & - & + & 39.12 & $<5$ \\
\hline 27 & + & + & + & 22.63 & 153,036 \\
\hline 28 & + & + & + & 22.35 & 186,114 \\
\hline 29 & + & + & + & 22.70 & 77,645 \\
\hline 30 & + & + & + & 33.46 & 34 \\
\hline 31 & + & + & + & 25.40 & 7,803 \\
\hline 32 & + & + & + & 22.93 & 41,974 \\
\hline 33 & + & + & + & 24.14 & 34,717 \\
\hline 34 & + & + & + & 24.20 & 33,609 \\
\hline 35 & + & + & + & 34.62 & $<5$ \\
\hline 36 & + & + & + & 23.12 & 67,130 \\
\hline 37 & + & + & + & 25.83 & 11,635 \\
\hline 38 & + & + & + & 28.97 & 2,089 \\
\hline 39 & + & + & + & 30.18 & 695 \\
\hline 40 & + & + & + & 23.40 & 55,991 \\
\hline 41 & + & + & + & 23.72 & 53,995 \\
\hline 42 & + & + & + & 25.05 & 19,265 \\
\hline 43 & + & + & + & 23.38 & 65,456 \\
\hline 44 & + & + & + & 22.11 & 132,134 \\
\hline 45 & + & + & + & 27.70 & 1,607 \\
\hline 46 & + & + & + & 26.60 & 3,070 \\
\hline 47 & + & + & + & 23.50 & 95,540 \\
\hline 48 & + & + & + & 23.20 & 117,119 \\
\hline 49 & + & + & + & 22.70 & 164,288 \\
\hline 50 & + & + & + & 24.10 & 70,637 \\
\hline 51 & + & + & + & 23.40 & 104,680 \\
\hline 52 & + & + & + & 23.10 & 127,212 \\
\hline 53 & + & + & + & 27.20 & 10,152 \\
\hline 54 & + & + & + & 23.30 & 107,239 \\
\hline 55 & + & + & + & 23.30 & 149,791 \\
\hline 56 & + & + & + & 23.0 & 136,542 \\
\hline 57 & + & + & + & 23.60 & 91,447 \\
\hline 58 & + & + & + & 25.20 & 33,250 \\
\hline 59 & + & + & + & 26.30 & 17,533 \\
\hline 60 & + & + & + & 22.0 & 251,430 \\
\hline 61 & + & + & + & 23.70 & 88,845 \\
\hline 62 & + & + & + & 23.40 & 102,788 \\
\hline 63 & + & + & + & 23.05 & 128,710 \\
\hline 64 & + & + & + & 23.60 & 102,443 \\
\hline 65 & + & + & + & 24.90 & 63,446 \\
\hline 66 & + & + & + & 24.60 & 48,246 \\
\hline 67 & + & + & + & 22.60 & 169,246 \\
\hline
\end{tabular}

${ }^{1}$ Cycle threshold.

${ }^{2}$ Not applicable. 
possibility to quantitate the number of copies present in the tested sample.

Regarding the specificity of the assay, the BLV SYBR $\mathrm{qPCR}$ was able to discern between true-positive and true-negative samples from an international reference panel. However, SYBR Green dye can bind to any amplified product producing a single amplification plot, and those amplification plots usually have the same appearance whether the amplification consists of target, nontarget, or a mixture of both. Considering that, the reaction specificity was further confirmed by dissociation curves analysis and electrophoresis in agarose gel. Additionally, no false-positive results were observed when several cell lines infected with different viruses other than BLV were tested by BLV SYBR qPCR. Altogether, these results highlight the high specificity of our in-house BLV qPCR.

Further, we evaluated the diagnostic specificity and sensitivity of the BLV SYBR qPCR using a set of 67 field samples, and the results were compared with those obtained by BLV nPCR and ELISA. A total of 3 discordant results were observed comparing all assays. The first one corresponded to 1 sample that tested negative by ELISA but positive by BLV nPCR and qPCR. Interestingly, this animal seroconverted in a later time point 2 mo apart. In contrast, none of the other sero-negative samples seroconverted during the same period of time. The combination of low levels of BLV proviral DNA and undetectable levels of BLV-specific antibodies in that particular sample might be indicative of recent BLV infection and could explain the discrepancy between serologic and molecular results observed for that particular animal. The second set of samples with discordant results was positive by ELISA and BLV SYBR qPCR but negative by BLV nPCR. Although both samples tested positive by BLV SYBR qPCR, we were not able to quantify the number of BLV copies in these samples because results fell below the dynamic range of the assay $(<5 \mathrm{BLV}$ copies per reaction). The discrepancy between both molecular tests could be explained by the presence of a very low number of copies of BLV proviral DNA in these samples and a higher sensitivity of BLV SYBR qPCR compared with nPCR. The failure of $\mathrm{nPCR}$ to detect some sero-positive animals with very low amounts of BLV provirus has been previously described by others (Eaves et al., 1994, Reichel et al., 1998). The high sensitivity of our BLV SYBR $\mathrm{qPCR}$ represents an important feature if the assay is intended to be used as screening tool because it would allow a higher proportion of BLV-infected animals to be detected as positive.

The transmission of BLV from cattle to humans is a controversial subject. In this regard, the production of medically important human recombinant proteins in milk might be a source of human exposure to BLV. Additionally, the screening of milk samples might be useful for any BLV eradication program due to their easy accessibility. For that reason, we analyzed the efficacy of our BLV SYBR qPCR to assess the presence or absence of BLV proviral DNA in a milk matrix (Kuckleburg et al., 2003; Gillet and Willems, 2016; Buehring et al., 2017). It has been demonstrated that a variety of inhibitors inherent to clinical and environmental matrices can decrease PCR sensitivity (i.e., proteases, complex polysaccharides, lipids, $\mathrm{Ca}^{++}$ions). Although the nucleic acid purification methods remove most of these inhibitors, some can co-elute with the DNA. Moreover, purification methods might result in low DNA recovery when applied to a complex matrix and further affect the sensitivity of the qPCR. In the present study, we demonstrated that neither the extraction method nor the BLV SYBR qPCR were affected by a milk matrix. This is important if the assay is intended to be used as screening for milk and milk-derived products.

\section{CONCLUSIONS}

Overall, the BLV SYBR qPCR described in this study is a sensitive and specific method for the detection of BLV proviral DNA. Due to its simplicity and low cost, it might be used as either a screening test or a confirmatory test for the clarification of inconclusive serological test results. Additionally, it can be used for detecting BLV proviral DNA in milk and milk subproducts, which are important sources of many transgenic proteins used in clinical settings.

\section{ACKNOWLEDGMENTS}

We thank the enzootic bovine leukemia reference laboratories that contributed to the development and characterization of the international panel of bovine leukemia virus proviral DNA samples: the Molecular and Cellular Biology Laboratory of Gemboux, University of Liege, Liege, Belgium; the Institute of Virology, Center for Infectious Diseases, University of Leipzig, Leipzig, Germany; the Department of Veterinary Medicine, Iwate University, Iwate, Japan; the National Veterinary Research Institute, Pulawy, Poland; and the Animal and Plant Health Agency, Weybridge, United Kingdom. We thank M. Rola-€uszczak and J. Kuzmak from the National Veterinary Research Institute for providing the plasmid bovine leukemia virus 1 (pBLV1) standard. The authors declare no conflicts of interest with respect to the research, authorship, and publication of this article. This work was supported by Concejo 
Nacional de Investigaciones Científicas y Tecnológicas (Buenos Aires, Argentina) and Instituto Nacional de Tecnología Agropecuaria (Buenos Aires, Argentina).

\section{REFERENCES}

Bartlett, P. C., L. M. Sordillo, T. M. Byrem, B. Norby, D. L. Grooms, C. L. Swenson, J. Zalucha, and R. J. Erskine. 2014. Options for the control of bovine leukemia virus in dairy cattle. J. Am. Vet. Med. Assoc. 244:914-922.

Buehring, G. C., H. Shen, D. A. Schwartz, and J. S. Lawson. 2017. Bovine leukemia virus linked to breast cancer in Australian women and identified before breast cancer development. PLoS One 12:e0179367.

Ciprian, F. 1973. Comprobacion de la leucemia bovina en Argentina. Revista Medica Veterinaria 54:2.

Eaves, F. W., J. B. Molloy, C. K. Dimmock, and L. E. Eaves. 1994. A field evaluation of the polymerase chain reaction procedure for the detection of bovine leukaemia virus proviral DNA in cattle. Vet. Microbiol. 39:313-321.

Frie, M. C., and P. M. Coussens. 2015. Bovine leukemia virus: A major silent threat to proper immune responses in cattle. Vet. Immunol. Immunopathol. 163:103-114.

Ghysdael, J., C. Bruck, R. Kettmann, and A. Burny. 1984. Bovine leukemia virus. Curr. Top. Microbiol. Immunol. 112:1-19.

Gillet, N. A., and L. Willems. 2016. Whole genome sequencing of 51 breast cancers reveals that tumors are devoid of bovine leukemia virus DNA. Retrovirology 13:75.

Gutiérrez, G., H. Carignano, I. Alvarez, C. Martinez, N. Porta, R. Politzki, M. Gammella, M. Lomonaco, N. Fondevila, M. Poli, and K. Trono. 2012. Bovine leukemia virus p24 antibodies reflect blood proviral load. BMC Vet. Res. 8:187.

Heenemann, K., S. Lapp, J. P. Teifke, D. Fichtner, T. C. Mettenleiter, and T. W. Vahlenkamp. 2012. Development of a bovine leukemia virus polymerase gene-based real-time polymerase chain reaction and comparison with an envelope gene-based assay. J. Vet. Diagn. Invest. 24:649-655.
Juliarena, M. A., C. N. Barrios, M. C. Ceriani, and E. N. Esteban 2016. Hot topic: Bovine leukemia virus (BLV)-infected cows with low proviral load are not a source of infection for BLV-free cattle. J. Dairy Sci. 99:4586-4589.

Kerkhofs, P., H. Heremans, A. Burny, R. Kettmann, and L. Willems. 1998. In vitro and in vivo oncogenic potential of bovine leukemia virus G4 protein. J. Virol. 72:2554-2559.

Kuckleburg, C. J., C. C. Chase, E. A. Nelson, S. A. Marras, M. A. Dammen, and J. Christopher-Hennings. 2003. Detection of bovine leukemia virus in blood and milk by nested and real-time polymerase chain reactions. J. Vet. Diagn. Invest. 15:72-76.

Lew, A. E., R. E. Bock, J. B. Molloy, C. M. Minchin, S. J. Robinson and P. Steer. 2004. Sensitive and specific detection of proviral bovine leukemia virus by $5^{\prime}$ Taq nuclease PCR using a $3^{\prime}$ minor groove binder fluorogenic probe. J. Virol. Methods 115:167-175.

OIE. 2012. Ezootic bovine leucosis. Pages 721-732 in Manual of Diagnostic Tests and Vaccines for Terrestrial Animals. 7th ed. World Organization of Animal Health, Paris, France.

Reichel, M. P., K. M. Tham, S. Barnes, and R. Kittelberger. 1998. Evaluation of alternative methods for the detection of bovine leukaemia virus in cattle. N. Z. Vet. J. 46:140-146.

Rola-Euszczak, M., C. Finnegan, M. Olech, B. Choudhury, and J. Kuzmak. 2013. Development of an improved real time PCR for the detection of bovine leukaemia provirus nucleic acid and its use in the clarification of inconclusive serological test results. J. Virol. Methods 189:258-264.

Trainin, Z., J. Brenner, R. Meirom, and H. Ungar-Waron. 1996. Detrimental effect of bovine leukemia virus (BLV) on the immunological state of cattle. Vet. Immunol. Immunopathol. 54:293-302.

Trono, K. G., D. M. Perez-Filgueira, S. Duffy, M. V. Borca, and C. Carrillo. 2001. Seroprevalence of bovine leukemia virus in dairy cattle in Argentina: Comparison of sensitivity and specificity of different detection methods. Vet. Microbiol. 83:235-248.

Yuan, Y., Y. Kitamura-Muramatsu, S. Saito, H. Ishizaki, M. Nakano, S. Haga, K. Matoba, A. Ohno, H. Murakami, S. N. Takeshima, and Y. Aida. 2015. Detection of the BLV provirus from nasal secretion and saliva samples using BLV-CoCoMo-qPCR-2: Comparison with blood samples from the same cattle. Virus Res. 210:248-254. 